\title{
Combined training (strength plus aerobic) potentiates a reduction in body fat but only functional training reduced low-density lipoprotein cholesterol in postmenopausal women with a similar training load
}

\author{
Fabrício Eduardo Rossi ${ }^{1,2 *}$, Ana Claudia S. Fortaleza', Lucas M. Neves ${ }^{3}$, Tiego A. Diniz', Marcela R. de Castro ${ }^{5}$, Camila Buonani6, \\ Jorge Mota ${ }^{7}$, Ismael F. Freitas Junior ${ }^{6}$
}

'Department of Physical Education, Federal University of Piaui, Teresina, Brazil

${ }^{2}$ Institute of Bioscience,São Paulo State University, Rio Claro, Brazil

${ }^{3}$ School of Physical Education and Sport, University of São Paulo, São Paulo, Brazil

${ }^{4}$ Department of Cell and Developmental Biology, Institute of Biomedical Sciences, University of São Paulo, São Paulo, Brazil

${ }^{5}$ Department of Physical Education, Federal University of Maranhão (UFMA), São Luis, Brazil

${ }^{6}$ Department of Physical Education, University Estadual Paulista, Presidente Prudente, Brazil

${ }^{7}$ Faculty of Sport Sciences and Physical Education, Porto University, Porto, Portugal

The aim of this study was to compare the effects of combined (CT; strength plus aerobic) and functional training (FT) on the body composition and metabolic profile with a similar training load in postmenopausal women. The participants were divided into three groups: $C T(n=20)$, FT $(n=17)$, and control group ( $C G, n=15)$. The trunk FM, fat mass (FM), percentage of FM (FM\%), and fat-free mass were estimated by dual-energy X-ray absorptiometry. The metabolic profile, glucose, triacylglycerol, total cholesterol, high-density lipoprotein cholesterol and low-density lipoprotein cholesterol (LDL-c) were assessed. There were main effects of time in trunk fat, $\mathrm{FM}$, and $\mathrm{FM} \%(P<0.05)$. There were statistically significant interaction for $\mathrm{FM}(P=0.015), \mathrm{FM} \%(P=0.017)$ with lower values for $C T$ group. For $L D L-c$, there was significant interaction $(P=0.002)$ with greater values for $\mathrm{FT}$ group in relation to $\mathrm{CG}$ and CT. Furthermore, when performed the post hoc test on the "mean absolute differences" $(\Delta)$, it can observed statistically significant difference between $\mathrm{FT}, \mathrm{CT}$, and CG $(-13.0 \pm 16.5 \mathrm{mg} / \mathrm{dL}$ vs. $4.8 \pm 18.4 \mathrm{mg} / \mathrm{dL}$ vs. $9.2 \pm 18.8 \mathrm{mg} / \mathrm{dL}, P<0.05)$. In conclusion, when training loads are equivalent $\mathrm{CT}$ potentiated a reduction in $\mathrm{FM}$ and FM\%, however, only FT reduced LDL-c in postmenopausal women.

Keywords: Aerobic training, Strength training, Body composition, Lipid profile

\section{INTRODUCTION}

The menopause involves psychological, hormonal and physical alterations (Stojanovska et al., 2014) which can lead to a marked reduction in lean body mass and an increase in total adipose and trunk fat (TF) (Sims et al., 2013). These changes contribute to reduced mobility and functionality (Bannerman et al., 2002), in addition to the development of cardiovascular diseases, type 2 diabe-

\footnotetext{
${ }^{*}$ Corresponding author: Fabricio Eduardo Rossi

(D) http://orcid.org/0000-0002-0594-2529

Department of Physical Education, Paulista State University "Júlio Mesquita Filho", Roberto Simonsen Street, n 305 - Educational Center, Presidente Prudente, 19060900, São Paulo, Brazil

Tel: +55-18-3229-5828, Fax: +55-18-3221-4391, E-mail: rossifabricio@yahoo.com.br Received: February 15, 2017 / Accepted: June 6, 2017
}

tes, hypertension, dyslipidemia, and metabolic syndrome (Kueht et al., 2009). On the other hand, physical activity is considered an important strategy for tackling these changes (Stojanovska et al., 2014), avoiding an increase in fat mass (FM) (Peterson et al., 2011; Sims et al., 2013), improvements in metabolic profile (Park et al., 2015) and quality of life (de Souza Santos et al., 2011).

Several studies from our group had showed the benefits of different kinds of training on the body composition and metabolic 
profile in postmenopausal women (Diniz et al., 2016; Neves et al., 2017; Rossi et al., 2013; Rossi et al., 2015; Rossi et al., 2016). Diniz et al. (2016) demonstrated that only 8 weeks of aerobic training prescribed by the critical velocity was sufficient in reduced total FM, percentage of fat and low-density lipoprotein cholesterol (LDL-c) in postmenopausal women. Rossi et al. (2013, 2015) showed that resistance plus aerobic training in the same session (combined training, CT) was effective in improving fatfree mass (FFM) and decreased whole and trunk adiposity after short period and post-16 weeks of training.

More recently, new programs of training have used at the literature, such as vibrating platforms (Emerenziani et al., 2014), elastic bands (Chang et al., 2012), and unstable bases (Chulvi-Medrano et al., 2012). Neves et al. (2017) investigated the effects of functional training (FT), which had used activities aimed at developing strength, endurance, agility, proprioception and neuromuscular control (Beckham and Harper, 2010) and observed improved on the body composition, lipid profile, and functional fitness in postmenopausal women, and suggested that the FT, using implements such as elastic bands, free weights and unstable bases could be an interesting strategies to improve healthy in postmenopausal women.

However, defining which strategy is the most effective in promoting changes in body composition constitutes a problem which has yet to be solved because the training protocols are compared without equal parameters concerning the quantification of training load (TL). One alternative that has been used to quantify TL is based on the training impulses (TRIMP), which it has been applied in recent studies (Akubat et al., 2012; Borresen and Lambert, 2008; Scott et al., 2013) and it is notable for its low cost and easy implementation.

Our group conducted a recently study and verified the effects of 16 weeks of aerobic and aerobic plus resistance training on the body composition and lipid profile of obese postmenopausal women and to analyzed which of these models were more effective after equalizing the TL using the TRIMP and demonstrated that CT potentiated a reduction in FM but there were no difference on the metabolic profile in postmenopausal women when a similar TL was used (Rossi et al., 2016).

Therefore, in order to ascertain the best training program to improve body composition and metabolic profile in postmenopausal women, and if FT is more effective than strength plus aerobic after equalizing the TL is not clear. Thus, this study aimed to compare the effects of 8 weeks of combined and FT on the body composition and metabolic profile with a similar TL in postmenopausal women. It was hypothesized that CT, as it contains ele- ments of traditional strength training (dumbbells, machines) would be the more effective in reducing FM, increase FFM as well as in improve metabolic profile than FT, using elastic bands, free weights and unstable bases.

\section{MATERIALS AND METHODS}

\section{Subjects}

Subjects were invited through television and newspaper advertising to participate in the study. The participants contacted the researchers by telephone and an appointment was made in order to carry out a more detailed interview. The inclusion criteria were: (a) postmenopausal women (having had no menstrual cycle for one or more years) (World Health Organization, 1996) and follicle-stimulating hormone $\geq 26.72 \mathrm{mUI} / \mathrm{mL}$; (b) not presenting any physical limitations or health problems that could prevent the completion of the assessments and exercise intervention (uncontrolled diabetes, hypertension, or rheumatoid arthritis); (c) presenting a medical certificate to participate in the training; (d) not having participated in any systematic physical exercise for, at least, 6 months prior to the study; (e) not receiving hormone replacement therapy. All participants signed the consent form and the present project was approved by the Ethics Research Group of the University (Protocol 64/2011).

Out of a total of 207 women who participated in the first screening, only 75 met all the inclusion criteria and agreed to participate in the study protocol. The subjects were randomly allocated into three groups: CT $(\mathrm{n}=25)$, FT $(\mathrm{n}=25)$, and control group (CG, $\mathrm{n}=25)$. The final sample consisted of 52 participants: CT $(n=20)$, FT $(n=17)$, and CG $(n=15)$. The reasons for dropouts included personal/family problems; unspecified reasons, absence of blood sample data or an accumulation of three consecutive absences or four nonconsecutive absences during 1 month.

\section{Procedures}

Evaluations were performed at baseline and after 8 weeks of training at the Science and Technology Department of the São Paulo State University (FCT/UNESP), Presidente Prudente Campus and involved screening for inclusion in the study, anthropometric, body composition and metabolic profile measurements.

The anthropometric measurements were conducted on the same morning as the dual-energy X-ray absorptiometry (DXA) scan, after a 4-hr fasting and subjects barefoot and wearing light clothing. Body composition was assessed between 8:00 a.m. and 12:00 p.m., by the same evaluator. Blood samples were collected after an 
overnight fast (12 hr).

Two weeks prior to evaluating, the participants performed one sets of 12-15 repetition in each exercise, 3 times per week (Monday, Wednesday, and Friday) for familiarization with equipment and training routine. The control group maintained 8 weeks of a sedentary lifestyle without participating in any regular physical exercise-essentially maintaining their habits. All training sessions were conducted under the supervision of physical education professionals.

\section{Anthropometric measurements and body composition}

Anthropometry was composed of body weight and height measurements. Height was measured on a fixed stadiometer of the Sanny brand, with an accuracy of $0.1 \mathrm{~cm}$ and length of $2.20 \mathrm{~m}$. Body weight was measured using an electronic scale (Filizola PL 50, Filizzola Ltda., Brazil), with a precision of $0.1 \mathrm{~kg}$. Body composition was estimated using a DXA scanner ver. 4.7 (Lunar DPX-NT, General Electric Healthcare, Buckinghamshire, England). The participants were positioned in the supine position and remained motionless throughout the examination. FM, percentage of FM (FM\%), FFM, and TF were assessed and expressed in absolute values by the DXA software. TF was estimated in the abdominal region, and was defined as $20 \%$ of the length from a circumference line at the pelvis to a circumference line at the neck (Bhupathiraju et al., 2011). All measurements were carried-out at the University laboratory, in a temperature controlled room. Each morning, before the beginning of the measurements, the equipment was calibrated by the same researcher, according to the manufacturer's instructions.

\section{Blood samples}

The blood samples were taken at the baseline and postintervention. After an overnight fast (12 hr), venous blood samples were collected to measure glucose $(\mathrm{mg} / \mathrm{dL})$ total fasting cholesterol (Chol, mg/dL), high-density lipoprotein cholesterol (HDL-c, mg/ $\mathrm{dL}$ ) using the colorimetric technique and dry chemicals, with equipment of the Johnson and Johnson brand, model Vitro 250. The Friedewald formula (Friedewald et al., 1972) was used to calculate LDL-c $(\mathrm{mg} / \mathrm{dL})$ concentration.

\section{Dietary intake assessment}

Twenty-four hour dietary recalls were conducted at 2-time points, on three nonconsecutive days (one weekend and two weekdays): 1 week before the beginning and in the first week after the intervention. The participants were oriented by a nutritionist as to how to complete the food records. Data were analyzed by the same nutritionist using the software NutWin, version 1.5 (Nutritional Program, Federal University of São Paulo, Brazil, 2002).

\section{CT procedures}

CT (strength plus aerobic) was performed by strength training plus aerobic training, including 10 min of warm-up and stretching at the end of the training session.

The determination of the intensity of the aerobic training was performed using the critical velocity protocol proposed by Wakayoshi et al. (1993) and used in our previous studies with postmenopausal women (Diniz et al., 2016; Neves et al., 2017). The studied group traveled three distances (400, 800, and 1,200 m) on a running track on separate, nonconsecutive days. The participants were instructed to cover the distance in the shortest possible time, which was recorded using a digital stopwatch (model S810i or RS800, Polar Electro, Espoo, Finland). The relationship between the distance $(\mathrm{m})$ and the exercise time (s) was linearly adjusted and the critical velocity was assumed to be the slope of this model (Zagatto et al., 2013) which is the intensity of aerobic training (Takahashi et al., 2009; Wakayoshi et al., 1993).

The resistance exercises used in the program were: $45^{\circ} \mathrm{leg}$ press, leg extension, leg curl, bench press, seated row, arm curl, triceps extension, side elevation with dumbbells, and abdominal exercises. The resistance training program consisted of two progressive phases: phase 1, 1st to 4th weeks, 12-15 repetitions, three sets per exercise, $60 \mathrm{sec}$ between sets; phase 2, 5th to 8th weeks, 10 12 repetitions, three sets per exercise, $60 \mathrm{sec}$ between sets. The TL was adjusted every four training weeks in order to maintain the prescribed number of repetitions (Table 1).

The intensity of the resistance training was controlled through the zone of maximum repetitions. The series were executed until momentary exhaustion, meaning that when participants performed the training with repetitions varying from 12 to 15 repetition maximum, they were always stimulated to execute at least 12 and no more than 15 repetitions. In the case of the participants executing more than 15 repetitions, the overload was increased in order to have the training zone respected (Rossi et al., 2015).

\section{FT procedures}

FT was performed by FT plus aerobic training, including 10 min of warm-up and stretching at the end of the training session. The intensity of the aerobic training was determined by the critical velocity, similar to the model described in the CT and applied in our previous study with FT and postmenopausal women (Neves et al., 2017). The FT was composed of 11 exercise stations devel- 
Rossi FE, et al. - Comparison of training with equalizing load.

Table 1. Summary of interventions

\begin{tabular}{|c|c|c|c|c|c|}
\hline Intervention & Exercise & Session details & $\begin{array}{l}\text { Session } \\
\text { time }\end{array}$ & $\begin{array}{c}\text { Rest } \\
\text { interval }\end{array}$ & Training load \\
\hline \multirow[t]{2}{*}{ Combined training } & Strength & 11 Exercises for strength training exercise & $40 \mathrm{~min}$ & $1 \mathrm{~min}$ & $\begin{array}{l}\text { Week } 1 \text { to } 4: 3 \times 12-15 \text { repetitions } 65 \%-70 \% 1 \text { RM each exercise (RPE 12-13) } \\
\text { Week } 5 \text { to } 8: 3 \times 10-12 \text { repetitions 70\%-75\% 1RM (RPE 12-13) }\end{array}$ \\
\hline & Aerobic & Walking in athletics track (400 m) & $30 \mathrm{~min}$ & None & $100 \%$ Critical velocity \\
\hline \multirow[t]{2}{*}{ Functional training } & Functional & $\begin{array}{l}11 \text { Exercises for strength training, agility, } \\
\text { coordination, and balance exercise }\end{array}$ & $40 \mathrm{~min}$ & $30 \mathrm{sec}$ & $\begin{array}{l}\text { Week } 1 \text { to } 4: 3 \text { series circuit - stimulus } 40 \text { sec each exercise (RPE 12-13) } \\
\text { Week 5-8: } 3 \text { circuit series - stimulus } 50 \text { sec each exercise (RPE 12-13) }\end{array}$ \\
\hline & Aerobic & Walking in athletics track (400 m) & $27-30 \mathrm{~min}$ & None & $100 \%$ Critical velocity \\
\hline
\end{tabular}

$\mathrm{RPE}$, rating of perceived exertion; 1RM, one-repetition maximum.

oped in circuit format, which the participants completed three times with a pause of $30 \mathrm{sec}$ between each station. At the end of the exercise the participants performed a 28- to 30-min walk, depending on the overload performed (Table 1).

The functional exercises were performed with elastic bands and free weights and consisted of sit-ups, arm curls, lateral raises, seated rows, knee flexions, crucifixes, handle triceps and squats. The agility drills were conducted using movement between cones, agility ladders were used for coordination and unstable bases such as a Bosu ball, swissball, boards and balance discs were used for balance exercises. There were also variations in the support base and movement of the upper body in the upright position. The exercises are illustrated in Fig. 1.

The FT program consisted of two progressive phases: phase 1, 1st to 4th weeks, 40 sec of execution and 30-sec pause between exercises; phase 2, 5th to 8th weeks, 50 sec of execution and 30sec pause between exercises. The training intensity was monitored by rating of perceived exertion (RPE), due to the variations in tension/overload of the equipment used (Table 1).

\section{Session RPE}

A previous pilot study was performed to equalize the load of the training groups, which involved a session of combined and FT in different day. For load equalization, the 20-point scale as standardized by Borg et al. (1987) was used to determine the RPE at the end of each training and the time of sessions was modified to induce similar values of RPE between groups [RPE $=13-14$ (arbitrary units) ${ }^{-1}$.

The pilot study indicated the necessity of $27 \mathrm{~min}$ of resistance exercises and $30 \mathrm{~min}$ of aerobic exercise for the CT. For FT, the pilot study indicated the necessity of $33 \mathrm{~min}$ of functional exercises and $27 \mathrm{~min}$ of aerobic exercise.

The monitoring impulse training (TRIMP) for each session was calculated by multiplying the duration of the session and the RPE (i.e., TRIMP [a.u.] = Duration [min] $\times$ RPE [arbitrary units $]^{-1}$ ) as proposed by Foster et al. (2001). This model was chosen because it
Strength

exercises

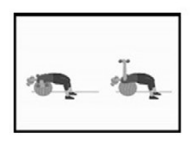

Exercise 1

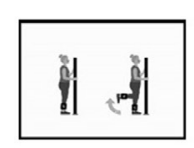

Exercise 3

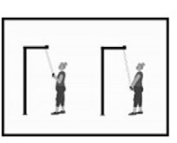

Exercise 5

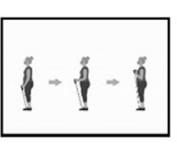

Exercise 7

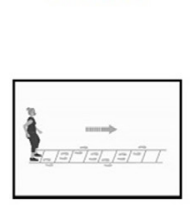

Coordination week 1 week 4

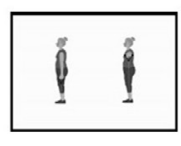

Exercise 2

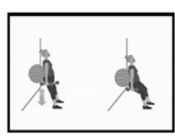

Exercise 4

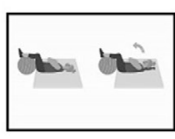

Exercise 6

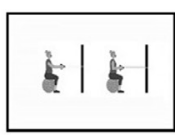

Exercise 8

Coordination

exercises

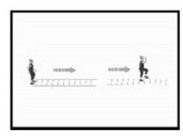

Coordination week 5 week 8

Balance

exercises

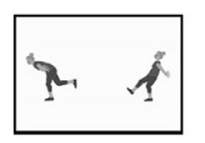

Balance - week 1 week 4

\section{Agility}

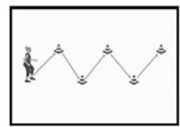

Agility - week 1 week 4

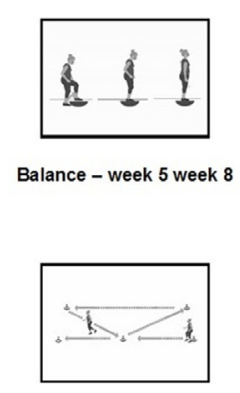

Agility - week 5 week 8

Fig. 1. Functional training program. 
allows the assessment of internal TL in both continuous efforts (i.e., aerobic stimuli) and intense efforts of short duration (i.e., resistance training). Thus, the TRIMP for the CT was taken as the sum of the impulses calculated for the strength training and the aerobic training (TRIMP [a.u.] $=\left[27 \times \mathrm{RPE}\right.$ of resistance $\left.{ }^{-1}\right]+[30 \times \mathrm{RPE}$ of aerobic-1]). The TRIMP for the FT was equivalent to the sum of the calculated impulses for the FT and aerobic training (TRIMP [a.u. $]=\left[33 \times \mathrm{RPE}\right.$ of functional $\left.{ }^{-1}\right]+\left[27 \times \mathrm{RPE}\right.$ of aerobic $\left.\left.\mathrm{c}^{-1}\right]\right)$.

The weekly TL was assumed as the average TRIMP observed in the three sessions per week, on condition that no significant difference was presented in the comparison between the two training models during the period (Table 2).

\section{Statistical analysis}

To compare the TRIMP between two training models the Mann-Whitney test was used and the median values and inter-

Table 2. Median values and interquartile range of the TRIMP per week

\begin{tabular}{llll}
\hline Moment & $\mathrm{FT}(\mathrm{n}=17)$ & $\mathrm{CT}(\mathrm{n}=20)$ & $P$-value \\
\hline Week 1 & $763.5(57)$ & $798.0(60)$ & 0.091 \\
Week 2 & $769.5(84)$ & $798.0(101)$ & 0.060 \\
Week 3 & $780.0(67)$ & $798.0(101)$ & 0.709 \\
Week 4 & $777.0(60)$ & $798.0(114)$ & 0.278 \\
Week 5 & $780.0(69)$ & $798.0(114)$ & 0.444 \\
Week 6 & $780.0(44)$ & $795.0(96)$ & 0.421 \\
Week 7 & $780.0(60)$ & $798.0(101)$ & 0.137 \\
Week 8 & $771.0(61)$ & $798.0(114)$ & 0.558 \\
\hline
\end{tabular}

Mann-Whitney test.

TRIMP, training impulses; $\mathrm{F}$, functional training; $\mathrm{CT}$, combined training. quartile range were presented. In the longitudinal analysis, the two-way analysis of variance (ANOVA) (time $\times$ group) was conducted. When a significant difference in group or interaction was observed, a Tukey post hoc test was conducted. Finally, the "mean absolute differences" (post exercise value minus baseline value $=\Delta$ ) were calculated and one-way ANOVA with Tukey Post hoc was used, which complementary analysis. The effect size (eta-squared; $\eta^{2}$ ) of each test was calculated for all analyses. All analysis was performed using the statistical software SPSS ver. 17.0 (SPSS Inc., Chicago, IL, USA) and the significance level adopted was $P<0.05$.

\section{RESULTS}

The weekly TRIMP behavior was not significantly different in the two experimental groups during the eight weeks (Table 2). In addition, the median of TRIMP during the training period also presented no significant differences between groups ( $\mathrm{FT}=6,203$ [381] a.u.; $C \mathrm{CT}=6,384$ [716]; $P=0.151$ ).

Table 3 presents the mean values of body weight, body composition, metabolic profile and dietary intake in the pre- and postintervention period in the three groups studied. In relation to the age $(\mathrm{CG}=61.9 \pm 7.2$ years; $\mathrm{FT}=60.1 \pm 5.4$ years; $\mathrm{CT}=62.2 \pm 6.3$ years; $P=0.575)$, high $(\mathrm{CG}=154.6 \pm 6.1 \mathrm{~cm} ; \mathrm{FT}=157.8 \pm 5.7 \mathrm{~cm}$; $\mathrm{CT}=155.8 \pm 5.3 \mathrm{~cm} ; P=0.274$ ) and all variables investigated, there were no statistical differences between groups at the baseline.

When performing the comparison on the body composition, there were main effects of time in trunk FM $(\mathrm{F}=8.003, P=0.007$, $\left.\eta^{2}=0.140\right), \mathrm{FM}\left(\mathrm{F}=16.401, P<0.001, \eta^{2}=0.25\right)$ and $\mathrm{FM} \%(\mathrm{~F}=$

Table 3. Comparison on the body composition, metabolic profile, and dietary intake in control group, functional, and combined training after 8 weeks

\begin{tabular}{|c|c|c|c|c|c|c|c|c|c|c|}
\hline \multirow{2}{*}{ Variable } & \multicolumn{3}{|c|}{$C G(n=15)$} & \multicolumn{3}{|c|}{$\mathrm{FT}(\mathrm{n}=17)$} & \multicolumn{3}{|c|}{ CT $(n=20)$} & \multirow{2}{*}{$\frac{\begin{array}{c}\text { Interaction } \\
\text { (time } \times \text { group) }\end{array}}{P \text {-value }}$} \\
\hline & Pre & Post & $\Delta$ & Pre & Post & $\Delta$ & Pre & Post & $\Delta$ & \\
\hline Weight (kg) & $61.4 \pm 8.4$ & $61.4 \pm 8.5$ & $-0.020 \pm 1.1$ & $64.1 \pm 8.5$ & $63.5 \pm 8.6$ & $-0.565 \pm 1.2$ & $59.7 \pm 5.8$ & $59.4 \pm 5.8$ & $-0.370 \pm 1.4$ & 0.469 \\
\hline $\mathrm{TF}(\mathrm{kg})$ & $14.0 \pm 3.2$ & $14.1 \pm 3.2$ & $0.077 \pm 0.6$ & $13.8 \pm 3.2$ & $13.3 \pm 3.2$ & $-0.467 \pm 0.5$ & $13.3 \pm 2.7$ & $12.8 \pm 2.8$ & $-0.489 \pm 0.9$ & 0.059 \\
\hline $\mathrm{FM}(\mathrm{kg})$ & $26.3 \pm 6.3$ & $26.3 \pm 6.3$ & $0.028 \pm 1.0$ & $26.1 \pm 5.2$ & $25.3 \pm 5.4$ & $-0.711 \pm 0.8$ & $24.9 \pm 4.8$ & $24.0 \pm 4.8^{*}$ & $-0.941 \pm 1.0^{\mathrm{a})}$ & 0.015 \\
\hline $\mathrm{FM}(\%)$ & $42.4 \pm 6.4$ & $42.4 \pm 6.3$ & $0.025 \pm 1.2$ & $40.4 \pm 4.0$ & $39.6 \pm 4.2$ & $-0.812 \pm 1.4$ & $41.5 \pm 5.5$ & $40.2 \pm 5.5^{*}$ & $-1.3 \pm 1.3^{\mathrm{a})}$ & 0.017 \\
\hline FFM (kg) & $33.0 \pm 4.5$ & $32.9 \pm 4.3$ & $-0.079 \pm 0.8$ & $35.7 \pm 4.0$ & $35.8 \pm 3.8$ & $0.164 \pm 1.5$ & $32.7 \pm 3.6$ & $33.3 \pm 3.5$ & $0.600 \pm 1.0$ & 0.230 \\
\hline Glucose (mg/dL) & $95.3 \pm 22.2$ & $94.6 \pm 18.8$ & $0.00 \pm 7.7$ & $96.9 \pm 17.8$ & $98.3 \pm 26.1$ & $1.4 \pm 15.0$ & $90.5 \pm 10.4$ & $88.8 \pm 10.6$ & $-1.7 \pm 6.8$ & 0.662 \\
\hline TAG (mg/dL) & $131.9 \pm 58.9$ & $128.6 \pm 57.0$ & $-3.3 \pm 35.1$ & $119.6 \pm 47.7$ & $110.3 \pm 45.5$ & $-9.3 \pm 36.1$ & $146.2 \pm 60.2$ & $128.9 \pm 53.0$ & $-17.3 \pm 62.7$ & 0.688 \\
\hline Chol (mg/dL) & $198.5 \pm 38.7$ & $209.2 \pm 41.6$ & $10.7 \pm 22.7$ & $198.5 \pm 35.8$ & $184.5 \pm 30.7$ & $-14.0 \pm 19.0^{a)}$ & $204.9 \pm 26.6$ & $205.2 \pm 21.3$ & $0.3 \pm 24.9$ & 0.079 \\
\hline HDL-c (mg/dL) & $51.1 \pm 8.6$ & $51.8 \pm 9.0$ & $0.7 \pm 3.7$ & $55.7 \pm 15.8$ & $56.6 \pm 14.1$ & $0.9 \pm 7.8$ & $55.5 \pm 14.6$ & $54.8 \pm 10.3$ & $-0.7 \pm 10.9$ & 0.822 \\
\hline LDL-c (mg/dL) & $121.5 \pm 30.6$ & $130.7 \pm 32.1$ & $9.2 \pm 18.8$ & $118.8 \pm 29.2$ & $105.8 \pm 26.7$ & $-13.0 \pm 16.5^{\mathrm{ab} b}$ & $120.2 \pm 20.8$ & $125.1 \pm 20.7$ & $4.8 \pm 18.4$ & 0.012 \\
\hline Dietary intake (kcal) & $1639.0 \pm 384.6$ & $1661.1 \pm 123.1$ & $22.0 \pm 297.9$ & $1529.6 \pm 347.2$ & $1634.3 \pm 260.5$ & $102.9 \pm 389.4$ & $1779.6 \pm 378.8$ & $1697.6 \pm 469.5$ & $-82.0 \pm 416.3$ & 0.477 \\
\hline
\end{tabular}

Values are presented as mean \pm standard deviation.

CG, control group; FT, functional training; CT, combined training; $\Delta$, mean absolute differences (post exercise value minus baseline value); TF, trunk fat; FM, fat mass; FFM, fat-free mass; TAG, triacylglycerol; Chol, total cholesterol; HDL-c, high-density lipoprotein cholesterol; LDL-c, low-density lipoprotein cholesterol.

${ }^{*}$ Statistic significant difference from pre. al $P<0.05$ compared to control group, Tukey post hoc test. ${ }^{b)} P<0.05$ compared to concurrent training, Tukey post hoc test with. 
14.305, $\left.P<0.001, \eta^{2}=0.23\right)$. There were significant interactions in FM and FM\% with lower values for CT group, according demonstrated on Table 3. In addition, post hoc analysis performed on the "mean absolute differences" $(\Delta)$ showed statistically significant difference between CT and CG group in FM $(P=0.013)$ and FM\% $(P=0.012)$. There were no main effects of time, group and interaction for TF and FFM $(P>0.05)$.

In relation to the metabolic profile, there were statistically significant interaction (time $\times$ group) for LDL-c $(\mathrm{F}=7.152, P=0.002$, $\left.\eta^{2}=0.23\right)$ with greater concentration for FT group. When performed the post hoc test on the "mean absolute differences" $(\Delta)$, it can observed statistically significant difference between FT and GC $(P=0.003)$ and FT and CT $(P=0.011)$. There were no main effect of time or significant differences between group and interaction for glucose, TAG and HDL-c $(P>0.05)$.

\section{DISCUSSION}

There is no consensus in the literature regarding the best training model for reducing body and TF and increasing FFM in postmenopausal women. Although studies have been conducted to find the most effective model, both in young people (Schwingshackl and Hoffmann, 2013) and middle-aged and elderly adults (Bales et al., 2012; Sanal et al., 2013; Seo et al., 2010), the majority of these studies made comparisons by standardizing only the total time of the training session, not carrying out appropriate load adjustments, and thus were unable to discern whether the changes in body composition were caused by the training stimulus or whether they were the consequence of the total quantity of work performed.

In the present study, when comparing the training protocols in the equalizing load model, it was observed that only 8 weeks of CT (strength plus aerobic) decreased FM and FM\%, however, FT was more effective in reduced LDL-c than CT and CG.

In accordance with these results, in previous study developed by our group (Rossi et al., 2016) verified the effects of isolated aerobic training and CT on the body composition and metabolic profile of postmenopausal women after equalizing the TLs using TRIMP and demonstrated that after 16 weeks both training programs induced an "antiatherogenic" profile, however, CT was more efficient in decreased FM and increased FFM. In this present study, although CT induced to increased FFM $(\Delta=0.600 \mathrm{~kg})$ there was not statistically significant difference in relation to the FT.

It is known that in women the period of menopause is associated with a reduction in FFM, and an increase in body fat, of up to
$2.5 \mathrm{~kg}$, principally in the trunk region (Sims et al., 2013). Excessive accumulation of central fat is considered the best predictor of cardiovascular risk, as it is associated with diabetes mellitus, hypertension and dyslipidemia, which contribute to the development of cardiovascular diseases (Kueht et al., 2009). In this sense the identification of effective measures to combat this situation are of great importance.

In relation to the metabolic profile, the improvement observed in the TF group, maybe have been favored the training format (circuit training). Several studies demonstrated benefits on metabolic profile during circuit training (Miller et al., 2014; Neves et al., 2017; Paoli et al., 2013).

Neves et al. (2017) verified the effect of circuit training format with eight stations related to the development of resistance force (using elastic bands for resistance) plus four stations focused on balance, coordination, and agility plus 18- to 30-min walk and observed that 16 weeks of FT performed using circuit training improved body composition, lipid profiles and functional fitness. However, a recent study has shown that the concurrent exercise can also promote the improvement in LDL-c (Atashak et al., 2016). Thus, despite there is no consensus at the literature regarding the intervention that produces greater improvements on metabolic profile, in this study TF group showed significant difference when compared to CG and CT for LDL-c.

It has been shown that a decrease of $1.0 \mathrm{mmol} / \mathrm{L}$ in $\mathrm{LDL}$ concentrations reduces by around 5\% the incidence of major vascular events (Cholesterol Treatment Trialists' (CTT) Collaboration et al. 2010). In this way, exercise program has been well established as a way to improves serum lipoprotein and lipid profiles and thereby, reduces cardiovascular risk (Lira et al., 2010). Indeed, a meta-analysis of 51 exercise training studies showed an average decrease of $3.7 \%$ in triacylglycerol and $5.0 \%$ in LDL-c, and an increment of 4.6\% in HDL-c (Leon and Sanchez, 2001), confirming the importance of exercise in the improvement of vascular health.

Despite these results being of great relevance, it is necessary to mention some limitations; there was no standardization of the diet, however the subjects maintained the same dietary intake during the study. In addition, it is suggested that other studies are needed which compare the effects of different physical exercise protocols, conducted over a longer period of time and using other variables, such strength and functional capacity.

In conclusion, when TLs are equivalent CT (strength plus aerobic) potentiated a reduction in FM and FM\%, however, only FT reduced LDL-c in postmenopausal women. 


\section{CONFLICT OF INTEREST}

No potential conflict of interest relevant to this article was reported.

\section{REFERENCES}

Akubat I, Patel E, Barrett S, Abt G. Methods of monitoring the training and match load and their relationship to changes in fitness in professional youth soccer players. J Sports Sci 2012;30:1473-1480.

Atashak S, Stannard SR, Azizbeigi K. Cardiovascular risk factors adaptation to concurrent training in overweight sedentary middle-aged men. J Sports Med Phys Fitness 2016;56:624-630.

Bales CW, Hawk VH, Granville EO, Rose SB, Shields T, Bateman L, Willis L, Piner LW, Slentz CA, Houmard JA, Gallup D, Samsa GP, Kraus WE. Aerobic and resistance training effects on energy intake: the STRRIDE-AT/RT study. Med Sci Sports Exerc 2012;44:2033-2039.

Bannerman E, Miller MD, Daniels LA, Cobiac L, Giles LC, Whitehead C, Andrews GR, Crotty M. Anthropometric indices predict physical function and mobility in older Australians: the Australian Longitudinal Study of Ageing. Public Health Nutr 2002;5:655-662.

Beckham SG, Harper M. Functional training: fad or here to stay? ACSM's Health Fit J 2010;14:24-30.

Bhupathiraju SN, Dawson-Hughes B, Hannan MT, Lichtenstein AH, Tucker KL. Centrally located body fat is associated with lower bone mineral density in older Puerto Rican adults. Am J Clin Nutr 2011;94: 1063-1070.

Borg G, Hassmén P, Lagerström M. Perceived exertion related to heart rate and blood lactate during arm and leg exercise. Eur J Appl Physiol Occup Physiol 1987;56:679-685.

Borresen J, Lambert MI. Quantifying training load: a comparison of subjective and objective methods. Int J Sports Physiol Perform 2008;3:1630.

Chang TF, Liou TH, Chen CH, Huang YC, Chang KH. Effects of elastic-band exercise on lower-extremity function among female patients with osteoarthritis of the knee. Disabil Rehabil 2012;34:1727-1735.

Cholesterol Treatment Trialists' (CTT) Collaboration, Baigent C, Blackwell L, Emberson J, Holland LE, Reith C, Bhala N, Peto R, Barnes EH, Keech A, Simes J, Collins R. Efficacy and safety of more intensive lowering of LDL cholesterol: a meta-analysis of data from 170,000 participants in 26 randomised trials. Lancet 2010;376:1670-1681.

Chulvi-Medrano I, Martínez-Ballester E, Masiá-Tortosa L. Comparison of the effects of an eight-week push-up program using stable versus unstable surfaces. Int J Sports Phys Ther 2012;7:586-594.

de Souza Santos CA, Dantas EE, Moreira MH. Correlation of physical ap- titude; functional capacity, corporal balance and quality of life (QoL) among elderly women submitted to a post-menopausal physical activities program. Arch Gerontol Geriatr 2011;53:344-349.

Diniz TA, Fortaleza AC, Rossi FE, Neves LM, Campos EZ, Freitas Junior IF. Short-term program of aerobic training prescribed using critical velocity is effective to improve metabolic profile in postmenopausal women. Sci Sports 2016;31:95-102.

Emerenziani GP, Meucci M, Gallotta MC, Buzzachera CF, Guidetti L, Baldari C. Whole body vibration: unsupervised training or combined with a supervised multi-purpose exercise for fitness? J Sports Sci 2014; 32:1033-1041.

Foster C, Florhaug JA, Franklin J, Gottschall L, Hrovatin LA, Parker S, Doleshal P, Dodge C. A new approach to monitoring exercise training. J Strength Cond Res 2001;15:109-115.

Friedewald WT, Levy RI, Fredrickson DS. Estimation of the concentration of low-density lipoprotein cholesterol in plasma, without use of the preparative ultracentrifuge. Clin Chem 1972;18:499-502.

Kueht ML, McFarlin BK, Lee RE. Severely obese have greater LPS-stimulated TNF-alpha production than normal weight African-American women. Obesity (Silver Spring) 2009;17:447-451.

Leon AS, Sanchez OA. Response of blood lipids to exercise training alone or combined with dietary intervention. Med Sci Sports Exerc 2001;33(6 Suppl):S502-515.

Lira FS, Yamashita AS, Uchida MC, Zanchi NE, Gualano B, Martins E Jr, Caperuto EC, Seelaender M. Low and moderate, rather than high intensity strength exercise induces benefit regarding plasma lipid profile. Diabetol Metab Syndr 2010;2:31.

Miller MB, Pearcey GE, Cahill F, McCarthy H, Stratton SB, Noftall JC, Buckle S, Basset FA, Sun G, Button DC. The effect of a short-term high-intensity circuit training program on work capacity, body composition, and blood profiles in sedentary obese men: a pilot study. Biomed Res Int 2014;2014:191797.

Neves LM, Fortaleza AC, Rossi FE, Diniz TA, Codogno JS, Gobbo LA, Gobbi S, Freitas IF Jr. Functional training reduces body fat and improves functional fitness and cholesterol levels in postmenopausal women: a randomized clinical trial. J Sports Med Phys Fitness 2017;57: 448-456.

Paoli A, Pacelli QF, Moro T, Marcolin G, Neri M, Battaglia G, Sergi G, Bolzetta F, Bianco A. Effects of high-intensity circuit training, low-intensity circuit training and endurance training on blood pressure and lipoproteins in middle-aged overweight men. Lipids Health Dis 2013;12:131.

Park SM, Kwak YS, Ji JG. The effects of combined exercise on health-related fitness, endotoxin, and immune function of postmenopausal women with abdominal obesity. J Immunol Res 2015;2015:830567.

Peterson MD, Sen A, Gordon PM. Influence of resistance exercise on lean 
body mass in aging adults: a meta-analysis. Med Sci Sports Exerc 2011;43:249-258.

Rossi FE, Buonani C, Viezel J, da Silva EP, Diniz TA, dos Santos VR, Fernandes RA, Freitas Jr IF. Effect of combined aerobic and resistance training in body composition of obese postmenopausal women. Motriz: Rev Educ Fis 2015;21:61-67.

Rossi FE, Buonani da Silva C, Viezel J, Gerosa Neto J, Araujo Fernandes R, Mota J, Freitas Jr IF. Efeitos do treinamento concorrente na composição corporal e taxa metabólica de repouso em mulheres na menopausa. Rev Port Ciênc Desporto 2013;13:12-22.

Rossi FE, Fortaleza AC, Neves LM, Buonani C, Picolo MR, Diniz TA, Kalva-Filho CA, Papoti M, Lira FS, Freitas Junior IF. Combined training (aerobic plus strength) potentiates a reduction in body fat but demonstrates no difference on the lipid profile in postmenopausal women when compared with aerobic training with a similar training load. J Strength Cond Res 2016;30:226-234.

Sanal E, Ardic F, Kirac S. Effects of aerobic or combined aerobic resistance exercise on body composition in overweight and obese adults: gender differences. A randomized intervention study. Eur J Phys Rehabil Med 2013;49:1-11.

Schwingshackl L, Hoffmann G. Long-term effects of low-fat diets either low or high in protein on cardiovascular and metabolic risk factors: a systematic review and meta-analysis. Nutr J 2013;12:48.

Scott TJ, Black CR, Quinn J, Coutts AJ. Validity and reliability of the session-RPE method for quantifying training in Australian football: a comparison of the CR10 and CR100 scales. J Strength Cond Res
2013;27:270-276

Seo DI, Jun TW, Park KS, Chang H, So WY, Song W. 12 weeks of combined exercise is better than aerobic exercise for increasing growth hormone in middle-aged women. Int J Sport Nutr Exerc Metab 2010; 20:21-26.

Sims ST, Kubo J, Desai M, Bea J, Beasley JM, Manson JE, Allison M, Seguin RA, Chen Z, Michael YL, Sullivan SD, Beresford S, Stefanick ML. Changes in physical activity and body composition in postmenopausal women over time. Med Sci Sports Exerc 2013;45:1486-1492.

Stojanovska L, Apostolopoulos V, Polman R, Borkoles E. To exercise, or, not to exercise, during menopause and beyond. Maturitas 2014;77: 318-323.

Takahashi S, Wakayoshi K, Hayashi A, Sakaguchi Y, Kitagawa K. A method for determining critical swimming velocity. Int J Sports Med 2009;3 0:119-123.

Wakayoshi K, Yoshida T, Udo M, Harada T, Moritani T, Mutoh Y, Miyashita M. Does critical swimming velocity represent exercise intensity at maximal lactate steady state? Eur J Appl Physiol Occup Physiol 1993;66:90-95.

World Health Organization. Investigaciones sobre la menopausia en los años noventa: informe de un Grupo Cient'ifico de la OMS.Ginebra: Organizaciòn Mundial de la Salud; 1996.

Zagatto AM, Kalva-Filho CA, Loures JP, Kaminagakura EI, Redkva PE, Papoti M. Anaerobic running capacity determined from the critical velocity model is not significantly associated with maximal accumulated oxygen deficit in army runners. Sci Sports 2013;28:e159-165. 